\title{
Prescrição e uso de metilfenidato na atenção infanto-juvenil: uma revisão integrativa
}

\author{
Prescription and use of methylphenidate in child and \\ adolescent care: an integrative review
}

\section{Prescripción y uso de metilfenidato en el cuidado de niños y adolescentes: una revisión integradora}

\author{
Maycon Hoffmann Cheffer ${ }^{1}$, Gabrieli Patricio Rissi ${ }^{2}$, Bianca Machado \\ Cruz Shibukawa ${ }^{3}$, Ieda Harumi Higarashi ${ }^{4}$
}

\begin{abstract}
1.Enfermeiro, Doutor, Programa de Pós-Graduação em Enfermagem, Universidade Estadual de Maringá, Maringá-PR, Brasil. Orcid: 0000-0002-9361-0152

2.Enfermeira, Doutoranda em Enfermagem, Programa de Pós-Graduação em Enfermagem, Universidade Estadual de Maringá, Maringá-PR, Brasil. Orcid: 0000-0002-1702-4004

3.Enfermeira, Doutoranda em Enfermagem, Programa de Pós-Graduação em Enfermagem, Universidade Estadual de Maringá, Maringá-PR, Brasil. Orcid: 0000-0002-7739-7881

4.Enfermeira, Doutora e Professora Adjunta, Programa de Pós-Graduação em Enfermagem, Universidade Estadual de Maringá, Maringá-PR, Brasil. Orcid: 0000-0002-4205-6841
\end{abstract}

\begin{abstract}
Resumo
Introdução. A prescrição e o consumo de metilfenidato aumentam a cada ano, principalmente entre crianças e adolescentes, para o tratamento do transtorno do déficit de atenção e hiperatividade. Objetivo. Discutir a prescrição e o uso de metilfenidato no contexto do cuidado infantil e adolescente, à luz das evidências disponíveis na literatura. Método. Revisão integrativa da literatura com levantamento bibliográfico nas bases de dados CINAHL, PubMed, Web of Science e SCOPUS, de artigos publicados entre 2015 e 2019, sobre a ocorrência da prescrição de metilfenidato, sua posologia e finalidade em crianças e adolescentes. Resultados. A amostra final foi composta por 17 artigos. Observou-se que $88 \%$ da amostra utilizava metilfenidato para tratamento do transtorno do déficit de atenção e hiperatividade, $64,7 \%$ era do sexo masculino e $58,8 \%$ correspondia ao período da infância. A liberação imediata do metilfenidato mostrou-se a mais prescrita $(41,2 \%)$ e a atomoxetina foi 0 medicamento mais utilizado para a suspensão do metilfenidato por efeitos colaterais $(29,4 \%)$. Conclusões. A utilização do metilfenidato no contexto da atenção infanto-juvenil revelou que os usuários apresentaram melhora rápida dos sintomas após a introdução do medicamento e que a sua descontinuação geralmente tem sido associada a efeitos adversos de origem física, emocional e comportamental, os quais tendem a desaparecer com a interrupção do fármaco. Unitermos. Metilfenidato; Transtorno do Déficit de Atenção com Hiperatividade; Criança; Adolescente
\end{abstract}

\footnotetext{
Abstract

Introduction. The prescription and consumption of methylphenidate has increased every year, especially among children and adolescents for the treatment of attention deficit hyperactivity disorder. Objective. To discuss the prescription and use of methylphenidate in the context of child and adolescent care considering the evidence available in the literature. Method. integrative bibliographic review with bibliographic research in the CINAHL, PubMed, Web of Science and SCOPUS databases, of articles published between 2015 and 2019, about the existence of methylphenidate prescription, its dose and purpose in children and adolescents. Results. the final sample consisted of 17 articles. It was observed that $88 \%$ of the sample used methylphenidate to treat attention deficit hyperactivity disorder, $64.7 \%$ was male and $58.8 \%$ corresponded to the period of childhood. Immediate release of
} 
methylphenidate was shown to be the most prescribed (41.2\%) and atomoxetine was the most widely used drug for methylphenidate discontinuation due to side effects (29.4\%). Conclusion. the use of methylphenidate in the context of child and adolescent care revealed that users experienced a rapid improvement in symptoms after the introduction of the drug and that its discontinuation has generally been associated with adverse effects of physical, emotional, and behavioral origin, which tend to disappear with the discontinuation of the drug. Keywords. methylphenidate; attention deficit disorder with hyperactivity; child; adolescent

\section{Resumen}

Introducción. La prescripción y el consumo de metilfenidato ha aumentado cada año, especialmente entre niños y adolescentes para el tratamiento del trastorno por déficit de atención con hiperactividad. Objetivo. Discutir la prescripción y el uso de metilfenidato en el contexto del cuidado de niños y adolescentes a la luz de la evidencia disponible en la literatura. Método. Revisión bibliográfica integradora con pesquisa bibliográfica en las bases de datos CINAHL, PubMed, Web of Science y SCOPUS, de artículos publicados entre 2015 y 2019, acerca de la existencia de prescripción de metilfenidato, su dosis y propósito en niños y adolescentes. Resultados. La muestra final consistió en 17 artículos. Se observó que el 88 \% de la muestra usaba metilfenidato para tratar el trastorno por déficit de atención e hiperactividad, $64.7 \%$ era hombre y 58.8 \% correspondía al período de la infancia. Se demostró que la liberación inmediata de metilfenidato fue la más prescrita $(41.2 \%)$ y la atomoxetina fue la droga más utilizada ante la interrupción de metilfenidato debido a los efectos secundarios (29.4 \%). Conclusión. El uso de metilfenidato en el contexto del cuidado de niños y adolescentes reveló que los usuarios experimentaron una mejoría rápida en los síntomas después de la introducción del medicamento y que su interrupción generalmente se ha asociado con efectos adversos de origen físico, emocional y conductual, que tienden a desaparecer con la interrupción de la droga.

Palabras clave. metilfenidato; trastorno por déficit de atención con hiperactividad; niño; adolescente

Trabalho realizado na Universidade Estadual de Maringá, Maringá-PR, Brasil.

\section{INTRODUÇÃO}

O Transtorno de Déficit de Atenção e Hiperatividade (TDAH) é um distúrbio neurocomportamental comum da infância, podendo afetar crianças e seu desempenho acadêmico, bem-estar e interações sociais. Suas características são impulsividade, hiperatividade, inquietude e falta de atenção inconsistentes com o nível de desenvolvimento ${ }^{1-5}$.

As estimativas de prevalência de TDAH em crianças e adolescentes são crescentes, com valores entre 0,9\% e 
$26,8 \%$. A medida em que crescem os diagnósticos de TDAH crescem também as intervenções de tratamento que são complexas e incluem intervenções sociais, psicológicas, comportamentais e farmacológicas 5 .

O tratamento do TDAH tem como medicação de primeira escolha o cloridrato de metilfenidato $(\mathrm{MPH})$, um agente estimulante do sistema nervoso central pertencente a classe terapêutica dos psicoestimulantes, comercialmente, é divulgado com diferentes nomes, sendo eles: Ritalina ${ }^{\circ}$, Ritalina $L A \circledast$ e Concerta $\AA$, administrados por via oral. $A$ Ritalina ${ }^{\circledR}(\mathrm{MPH}$ - LI) apresenta-se sob a forma farmacêutica de comprimidos de liberação imediata, a Ritalina LA® (MPH - LM) como cápsulas de liberação modificada e o Concerta ${ }^{\circledR}$ (MPH - OROS) como comprimidos de liberação prolongada, liberado de maneira lenta no organismo por conta de um sistema osmótico 5 .

$\mathrm{Na}$ Europa, um desenho transversal revelou o aumento da prevalência de medicamentos para tratamento do TDAH com predomínio em crianças de 10 a 14 anos, tal aumento também revelou discrepâncias entre os países, foram de 1,8 para 3,9\% na Holanda (aumento de $111,9 \%$ ), de 1,3 a 2,2\% na Alemanha $(+62,4 \%)$, de 0,4 a $1,5 \%$ na Dinamarca $(+302,7 \%)$ e de 0,3 a $0,5 \%$ no Reino Unido $(+56,6 \%)^{2,5}$. Dados que podem evidenciar um aumento mundial na utilização de medicamentos para tratamento do TDAH.

$\mathrm{O}$ uso de metilfenidato tem aumentado gradativamente desde 2004, seis eventos envolvendo à sua segurança foram elencados: em 2006, a Agência Francesa de Segurança de 
Medicamentos e Produtos para Saúde (ANSM) orientou o monitoramento de farmacovigilância e toxicodependência. Em 2007, a Agência Europeia de Medicamentos (EMA) iniciou uma revisão sobre segurança. Em 2009 a EMA declarou que os benefícios continuaram a superar os riscos, porém, mantendo a vigilância dos riscos a longo prazo ${ }^{6-7}$.

Em 2011, a farmacovigilância francesa publicou uma carta sobre possíveis efeitos a longo prazo. Em 2012, a França reavaliava medicamentos contendo metilfenidato por concluírem que ainda existiam incertezas quanto aos efeitos de médio a longo prazo, principalmente efeitos cardiovasculares, neurológicos e psiquiátricos, e que havia risco de uso não médico, mau uso e abuso. Em 2013, a ANSM publicou um relatório para profissionais de saúde, a fim de garantir a prescrição de metilfenidato desde que os consumidores e familiares fossem informados dos riscos ${ }^{7}$.

O consumo de metilfenidato está relacionado à iniciação da infância e seu tratamento farmacológico consistente com as autorizações de sua comercialização para tratamento de criança e adolescentes com TDAH ${ }^{5,7}$.

Em revisão sistemática de ensaios clínicos randomizados os efeitos adversos apresentados ao uso de metilfenidato são transtornos psicóticos, arritmia, insônia, dificuldade em adormecer, problemas do sono, diminuição do apetite, dor de cabeça e dor abdominal. Não é possível estimar com precisão o risco real dos eventos adversos, no entanto, os eventos adversos graves, são menores do que a 
proporção de eventos adversos não graves os quais frequentemente levam à suspensão do metilfenidato ${ }^{8}$.

Existem tratamentos não farmacológicos para o TDAH que incluem neuro feedback, treinamento cognitivo, terapia cognitivo-comportamental, treinamento de crianças ou pais, suplementação dietética de ácidos graxos ômega e abordagens fitoterápicas e dietéticas. Apesar do amplo uso dessas abordagens, existem lacunas significativas sobre a não eficácia comparativa dos tratamentos não farmacológicos para o TDAH9.

Com o intuito de compreender o aumento do consumo de metilfenidato e sua repercussão no contexto da assistência pediátrica, objetivou-se discutir a prescrição e a utilização do metilfenidato no contexto da atenção infantojuvenil à luz das evidências disponíveis na literatura.

\section{MÉTODO}

Trata-se de uma revisão integrativa da literatura. Este método permite a investigação mais abrangente e profunda a respeito de uma determinada temática, por meio da síntese e conciliação de diferentes metodologias, contribuindo ativamente na prática baseada em evidência ${ }^{10}$.

Para seu desenvolvimento, realizou-se de maneira ordenada as seis etapas: 1) elaboração da questão de pesquisa; 2) busca e amostragem na literatura; 3) extração dos dados; 4) análise crítica dos estudos incluídos; 5) síntese 
e discussão dos resultados; e 6) apresentação da revisão integrativa ${ }^{11}$.

A questão de pesquisa elencada para nortear o estudo foi: "Como tem sido prescrito e utilizado o metilfenidato na atenção infanto-juvenil?". Com o propósito de responder à questão norteadora, realizou-se um levantamento bibliográfico nas bases de dados CINAHL (Cumulative Index to Nursing and Allied Health Literature), PubMed (National Library of Medicine), Web of Science e SCOPUS. A busca nas bases de dados ocorreu nos meses de março a abril de 2020.

Os descritores utilizados para busca das publicações foram os termos do Medical Subject Headings (MeSH), sendo eles "Methylphenidate", "Medicalization", "Amphetamine", "Central Nervous System Stimulants", "Child" e "Adolescent". Os descritores foram combinados em cada base de dados por meio dos operadores boleanos AND e OR.

Com a finalidade de ampliar e aprofundar a extração dos dados, realizou-se o cruzamento dos descritores supracitados, resultando em três etapas de busca em cada base de dados, sendo elas: Etapa 1: Methylphenidate AND Medicalization AND Child OR Adolescent. Etapa 2: Methylphenidate AND Amphetamine AND Child OR Adolescent. Etapa 3: Methylphenidate AND Central Nervous System Stimulants AND Child OR Adolescent.

Os critérios de inclusão das produções científicas foram: o período de publicação de 2015 a 2019, recorte temporal a partir de 2015 devido ao grande quantitativo de manuscritos publicados e a atualidade dos mesmos, até 2019 para 
contemplar o período sem novas publicações; constar no resumo a ocorrência da prescrição de metilfenidato, sua posologia e finalidade; não haver interrupção do tratamento; apresentação dos resultados da utilização no contexto da atenção infanto-juvenil com indivíduos de até 19 anos, ser artigo original e estar publicado nos idiomas português, inglês ou espanhol. A escolha pelo intervalo temporal buscou compreender um período significativo e atualizado, considerando a quantidade e representatividade das publicações.

Os critérios de exclusão compreenderam trabalhos no modelo de estudo experimental, estudos retrospectivos, estudos no formato de tese, dissertação, livro ou capítulo de livro, editorial, matéria de jornal, revisão integrativa ou sistemática da literatura, estudo reflexivo e outros estudos que não contemplassem o objetivo proposto na pesquisa.

Com o intuito de sistematizar a avaliação dos artigos selecionados, extraíram-se as informações para uma tabela previamente construída no software Microsoft Excelß, versão 2016.

Ressalta-se que a análise crítica dos estudos incluídos, a sua interpretação, discussão dos resultados e apresentação da síntese de conhecimento foram separados por sexo devido aos efeitos adversos provocados pelo uso do metilfenidato nos diferentes órgãos reprodutores. 
Dessa maneira, foi possível construir o fluxograma a seguir (Figura 1), o qual ilustra a seleção dos artigos, o que resultou em uma amostra de 17 artigos.

Figura 1. Fluxograma da seleção de artigos nas bases de dados Cinahl, PubMed, Scopus e Web of Science, conforme os critérios de inclusão e exclusão.

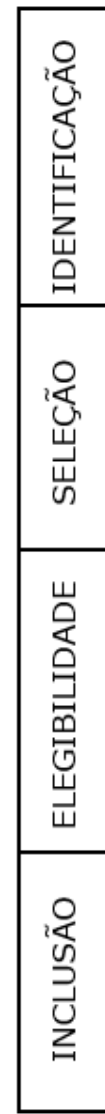

Estudos identificados nas fontes de informações CINAHL $(n=232)$, Scopus $(n=$ 974), Web of Science $(n=241)$, PubMed $(n=2728)$

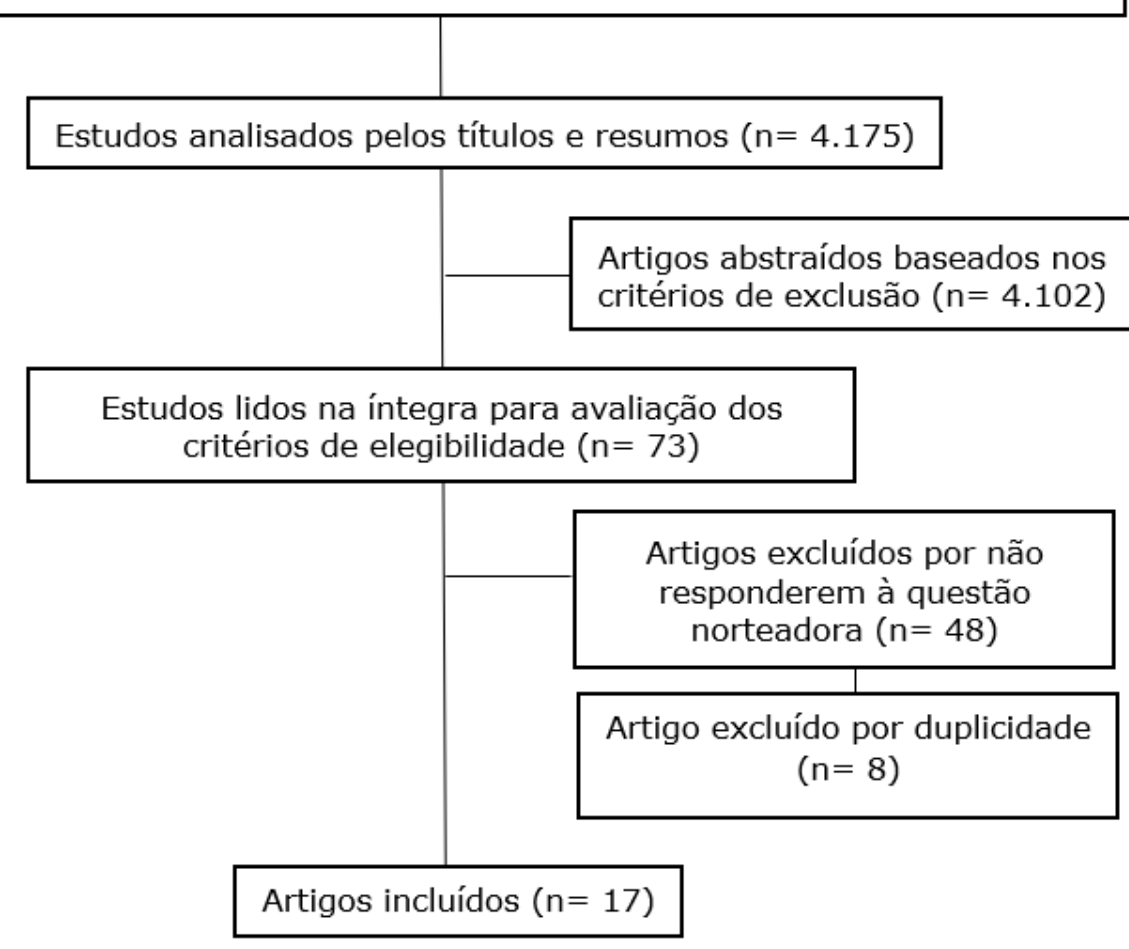

Para facilitar a análise e interpretação dos resultados, optou-se por determinar o grau de evidência dos artigos selecionados, utilizando os critérios apresentados na Figura 2, baseados no estudo realizado por Ribeiro (2019) ${ }^{12}$. 
Figura 2. Descrição dos Níveis de Evidências.

\begin{tabular}{|c|l|}
\hline \multicolumn{2}{|c|}{ Nível de Evidência } \\
\hline I & Evidências oriundas de sínteses de estudos de coorte ou caso-controle. \\
\hline II & Evidências derivadas de um único estudo de coorte ou caso-controle. \\
\hline III & Evidências obtidas de metassíntese ou síntese de estudos descritivos. \\
\hline IV & Evidências provenientes de estudos descritivos ou qualitativos. \\
\hline V & Evidências oriundas de opinião de especialistas. \\
\hline
\end{tabular}

Da amostra final, verificou-se que todos os estudos selecionados eram de caráter descritivo. Diante disso, o nível de evidência IV determinou a classificação de todos os artigos elegidos, o qual corresponde a evidências derivadas de estudos descritivos ou qualitativos.

Averiguou-se que o ano de 2015 representou 35,3\% das publicações, 2016 apresentou 23,6\%, 2017 apontou 17,6\%, 2018 exibiu a menor porcentagem, correspondendo a 5,9\% e, 2019 representou 17,6\% de todas as publicações. Observou-se que a maioria dos estudos foram desenvolvidos na Turquia (82\%), com a prevalência do idioma inglês.

\section{RESULTADOS}

Totalizaram-se 4175 artigos provenientes da busca inicial (Figura 3), dos quais foram lidos os títulos e resumos, resultando em 73 estudos elegíveis. Após a leitura integral deles, selecionou-se 25 artigos que atenderam aos critérios de inclusão e exclusão. Constatou-se que três trabalhos se encontravam na base de dados CINAHAL, nove na PUBMED 
e 13 na SCOPUS. Excluíram-se oito artigos que eram duplicados, resultando em uma amostra final de 17 estudos.

Figura 3. Sistematização da busca eletrônica nas bases de dados Cinahl, PubMed, Scopus e Web of Science.

\begin{tabular}{|l|c|c|c|c|}
\hline \multicolumn{1}{|c|}{ Descritores } & Cinahl & PubMed & Scopus & $\begin{array}{c}\text { Web of } \\
\text { Science }\end{array}$ \\
\hline $\begin{array}{l}\text { Methylphenidate AND Medicalization AND } \\
\text { Child OR Adolescent }\end{array}$ & 07 & 37 & 05 & 01 \\
\hline $\begin{array}{l}\text { Methylphenidate AND Amphetamine AND } \\
\text { Child OR Adolescent }\end{array}$ & 35 & 1747 & 329 & 200 \\
\hline $\begin{array}{l}\text { Methylphenidate AND Central Nervous } \\
\text { System Stimulants AND Child OR Adolescent }\end{array}$ & 190 & 944 & 640 & 40 \\
\hline Total & $\mathbf{2 3 2}$ & $\mathbf{2 7 2 8}$ & $\mathbf{9 7 4}$ & $\mathbf{2 4 1}$ \\
\cline { 2 - 6 } & $\mathbf{4 1 7 5}$ \\
\hline
\end{tabular}

Com a finalidade de melhorar a visualização dos artigos selecionados, as principais características e resultados foram divididos por sexo dos participantes que tiveram seu caso relatado e compilados nas Figuras 4 e 5.

Os resultados permitiram observar que a maioria ( $88 \%)$ dos artigos que compuseram a amostra final apresentaram o uso do Metilfenidato (MPH) como tratamento para o Transtorno do Déficit de Atenção com Hiperatividade (TDAH).

No que tange ao sexo dos participantes do estudo $(64,7 \%)$ era masculino, e o ciclo de vida com a prevalência $(58,8 \%)$ da amostra correspondia ao período da infância. Apenas uma criança se encontrava em idade pré-escolar, com 5 anos e 10 meses, sendo que o restante correspondeu a idade escolar, até 11 anos, 11 meses e 29 dias. 
Figura 4. Descrição da amostra por sexo masculino.

\begin{tabular}{|c|c|c|}
\hline $\begin{array}{l}\text { Ano/ } \\
\text { País/ } \\
\text { Base }\end{array}$ & $\begin{array}{c}\text { Autores/ } \\
\text { Tipo de estudo }\end{array}$ & Diagnóstico, prescrição e desfechos \\
\hline $\begin{array}{l}2017 \\
\text { Turquia } \\
\text { CINAHL/ } \\
\text { SCOPUS }\end{array}$ & $\begin{array}{l}\text { Arslan, Arslan, } \\
\text { Kilinç, et al. }{ }^{13} \\
\text { Relato de Caso }\end{array}$ & $\begin{array}{l}9 \text { anos, diagnóstico de TDAH. Usou } 18 \mathrm{mg} / \mathrm{kg} \text { de MPH-OROS. Apresentou discinesia } \\
\text { orofacial e de extremidades após a administração da primeira dose de MHP-OROS. } \\
\text { O medicamento foi descontinuado e a discinesia resolvida em } 20 \text { minutos após a } \\
\text { injeção intravenosa de Biperideno. }\end{array}$ \\
\hline $\begin{array}{l}2017 \\
\text { Turquia } \\
\text { CINAHL/ } \\
\text { SCOPUS }\end{array}$ & $\begin{array}{c}\text { Gunes }^{14} \\
\text { Relato de Caso }\end{array}$ & $\begin{array}{l}8 \text { anos, diagnóstico de Autismo e TDAH. Usou } 20 \mathrm{mg} \text { de MPH-LM e posteriormente } \\
10 \mathrm{mg} \text {. Um mês usando MPH } 20 \mathrm{mg} \text { os sintomas melhoraram parcialmente, mas } \\
\text { começou a apresentar Tricotilomania (TTM). A medicação foi cessada e a TTM foi } \\
\text { resolvida. Duas semanas depois para reavaliar foi introduzido MPH } 10 \mathrm{mg} \text { e a TTM } \\
\text { ressurgiu na primeira semana. }\end{array}$ \\
\hline $\begin{array}{l}2015 \\
\text { Reino } \\
\text { Unido } \\
\text { PUMED/ } \\
\text { SCOPUS }\end{array}$ & $\begin{array}{l}\text { Snell, Bakshi }{ }^{15} \\
\text { Relato de Caso }\end{array}$ & $\begin{array}{l}7 \text { anos, diagnóstico de TDAH. Usou MPH-LI } 10 \mathrm{mg} \text { duas vezes ao dia. Após três } \\
\text { meses apresentou marcha atáxica, discinesias orofaciais e coreoatetose dos } \\
\text { membros. Ficou hospitalizado e foi diagnosticado com meningoencefalite por efeito } \\
\text { adverso do MPH. A interrupção do MPH levou a melhora rápida e significativa dos } \\
\text { efeitos adversos neurológicos. }\end{array}$ \\
\hline $\begin{array}{l}2018 \\
\text { Turquia } \\
\text { PUMED }\end{array}$ & $\begin{array}{l}\text { Bilaç, Kutuk, } \\
\quad \text { Bilaç } 16 \\
\text { Relato de Caso }\end{array}$ & $\begin{array}{l}12 \text { anos, diagnóstico de TDAH. Iniciou MPH-LI } 36 \mathrm{mg} \text { ao dia. Com um mês houve } \\
\text { melhora dos sintomas do TDAH. Mas, após duas semanas teve alopecia difusa, } \\
\text { sendo associada ao efeito do MPH o qual foi descontinuado e a alopecia se } \\
\text { resolveu. Os sintomas do TDAH retornaram e Atomoxetina foi prescrito. }\end{array}$ \\
\hline $\begin{array}{l}2015 \\
\text { Turquia } \\
\text { PUMED }\end{array}$ & $\begin{array}{l}\text { Oncu, Colak, } \\
\text { Okan }{ }^{17} \\
\text { Relato de Caso }\end{array}$ & $\begin{array}{l}16 \text { anos, diagnóstico de TDAH. Usou MPH-LI } 30 \mathrm{mg} / \text { dia. Houve melhora na } \\
\text { concentração, mas apresentou sonolência e ejaculação espontânea com crescente } \\
\text { ansiedade. Nem ereções salientes nem excitação sexual foram associadas. Após } \\
\text { a cessação do MPH as ejaculações desapareceram. }\end{array}$ \\
\hline $\begin{array}{l}2016 \\
\text { Turquia } \\
\text { PUMED }\end{array}$ & $\begin{array}{l}\text { Ekinci, Direk, } \\
\text { Ekinci, et al. }{ }^{18} \\
\text { Relato de Caso }\end{array}$ & $\begin{array}{l}17 \text { anos, diagnóstico de lesão cerebral traumática e déficit de atenção. Prescrito } \\
18 \mathrm{mg} / \text { dia de MPH-OROS. Apresentou dificuldades de atenção nos trabalhos } \\
\text { escolares e esquecimento, após o trauma. Com uma semana de uso do MHP-OROS } \\
\text { apresentou queixas de conversar consigo mesmo, pensamentos delirantes, } \\
\text { irritabilidade e insônia. O MPH foi interrompido e os sintomas cessaram. }\end{array}$ \\
\hline $\begin{array}{l}2019 \\
\text { Turquia } \\
\text { SCOPUS }\end{array}$ & $\begin{array}{l}\text { Ekinci, } \\
\text { Ustundag, } \\
\text { Tunali, et al. }{ }^{19} \\
\text { Relato de Caso }\end{array}$ & $\begin{array}{l}5 \text { anos, diagnóstico de TDAH. Usou } 10 \mathrm{mg} / \mathrm{dia} \text { de MPH-LI. Após uso de MPH, teve } \\
\text { episódios de priapismo diariamente, acompanhado de dor e choro. O MPH foi } \\
\text { interrompido e o priapismo resolvido em uma semana. Depois disso, iniciou-se } \\
\text { atomoxetina, resolvendo o priapismo e o TDAH. }\end{array}$ \\
\hline $\begin{array}{l}2019 \\
\text { Turquia } \\
\text { SCOPUS }\end{array}$ & $\begin{array}{l}\text { Aksu, Akdere, } \\
\text { Toros }{ }^{20} \\
\text { Relato de Caso }\end{array}$ & $\begin{array}{l}7 \text { anos, diagnóstico de TDAH. Usou } 10 \mathrm{mg} \text { de MPH-LM duas vezes ao dia. Após dois } \\
\text { meses, duas lesões despigmentadas apareceram no joelho esquerdo. Não possuía } \\
\text { história familiar de vitiligo ou doença autoimune. Em exame dermatológico, as } \\
\text { lesões foram consideradas vitiligo associado ao uso de MPH. O tratamento foi } \\
\text { interrompido e foi acompanhado por TDAH sem medicação. Após três meses não } \\
\text { apresentou alterações nas lesões dermatológicas. }\end{array}$ \\
\hline $\begin{array}{l}2017 \\
\text { Turquia } \\
\text { SCOPuS }\end{array}$ & $\begin{array}{l}\text { Ercan, Inci, } \\
\text { Ipci } 21 \\
\text { Relato de Caso }\end{array}$ & $\begin{array}{l}13 \text { anos, diagnóstico de TDAH. Usou } 10 \mathrm{mg} / \text { dia de MPH-LM. No primeiro mês os } \\
\text { sintomas do TDAH foram controlados. Após quatro semanas, teve diminuição de } \\
\text { plaquetas de } 152 \times 103 / \mathrm{mm} 3 \text { para } 131 \times 103 / \mathrm{mm} 3 \text {. O MPH foi descontinuado e a } \\
\text { contagem voltou ao normal. Após } 20 \text { dias, o MPH foi reiniciado e a contagem de } \\
\text { plaquetas caiu novamente. O MPH foi interrompido e as plaquetas normalizaram. }\end{array}$ \\
\hline $\begin{array}{c}2016 \\
\text { Turquia } \\
\text { SCOPUS }\end{array}$ & $\begin{array}{c}\text { Akaltun }{ }^{22} \\
\text { Relato de Caso }\end{array}$ & $\begin{array}{l}14 \text { anos, diagnóstico de TDAH. Usou } 27 \mathrm{mg} / \mathrm{dia} \text { de MPH-OROS. Após duas } \\
\text { semanas, houve diminuição nos níveis de testosterona. Devido ao histórico de } \\
\text { testículo não descido, a testosterona era verificada todos os anos. O tratamento } \\
\text { com MPH foi interrompido e o nível de testosterona foi testado com achados } \\
\text { normais. Iniciou-se atomoxetina e a testosterona permaneceu sem alterações. }\end{array}$ \\
\hline $\begin{array}{c}2015 \\
\text { Turquia } \\
\text { SCOPUS }\end{array}$ & $\begin{array}{c}\text { Alpaslan, } \\
\text { Coskun, Kocak, } \\
\text { et al. }{ }^{23} \\
\text { Relato de Caso }\end{array}$ & $\begin{array}{l}7 \text { anos, diagnóstico de TDAH. Usou } 10 \mathrm{mg} / \mathrm{dia} \text { de MPH-LI. Sem histórico familiar de } \\
\text { gagueira, após } 10 \text { dias passou a apresentar prolongamentos sonoros, bloqueio } \\
\text { silencioso, produção de palavras com tensão física excessiva e repetições } \\
\text { monossilábicas de palavras inteiras. A gagueira foi associada ao MPH, o qual foi } \\
\text { descontinuado, sendo que em uma semana a fala voltou ao normal. Os sintomas } \\
\text { de TDAH reapareceram e foi prescrito atomoxetina. }\end{array}$ \\
\hline
\end{tabular}


Figura 5. Descrição da amostra por sexo feminino.

\begin{tabular}{|c|c|c|}
\hline $\begin{array}{l}\text { Ano/ } \\
\text { País/ } \\
\text { Base }\end{array}$ & $\begin{array}{l}\text { Autores/ } \\
\text { Tipo de } \\
\text { estudo }\end{array}$ & Diagnóstico, prescrição e desfechos \\
\hline $\begin{array}{c}2015 \\
\text { Turquia } \\
\text { CINAHL }\end{array}$ & $\begin{array}{l}\text { Mutlu, Bahali, } \\
\text { Gunes, et } \\
\text { al. }{ }^{24} \\
\text { Relato de } \\
\text { Caso }\end{array}$ & $\begin{array}{l}17 \text { anos, diagnóstico de TDAH e Retardo Mental Leve. De } 27 \mathrm{mg} \text { de MPH- } \\
\text { OROS passou a } 37 \mathrm{mg} / \mathrm{kg} \text {. Com o aumento da dose houve melhora } \\
\text { mínima nos sintomas do TDAH, mas a duração do ciclo menstrual } \\
\text { aumentou em } 15 \text { dias. Ao retornar para } 27 \mathrm{mg} \text { de MPH-OROS o ciclo } \\
\text { menstrual normalizou e o TDAH obteve melhora. }\end{array}$ \\
\hline $\begin{array}{l}2015 \\
\text { Taiwan } \\
\text { PUBMED/ } \\
\text { SCOPUS }\end{array}$ & $\begin{array}{l}\text { Hu, Lin, } \\
\text { Chang, et } \\
\text { al. } .^{25} \\
\text { Relato de } \\
\text { Caso }\end{array}$ & $\begin{array}{l}16 \text { anos, diagnóstico de ataxia cerebelar e TDAH. Prescrito } 5 \mathrm{mg} \text { de MPH- } \\
\text { LI duas vezes ao dia. Apresentou início agudo de fácil distração, } \\
\text { hiperatividade, diminuição do sono e comportamentos interpessoais } \\
\text { distorcidos. Após uso de MPH apresentou melhora dos sintomas do } \\
\text { TDAH. No primeiro dia já houve melhora da desatenção e } \\
\text { comportamentos impulsivos. }\end{array}$ \\
\hline $\begin{array}{c}2019 \\
\text { Turquia } \\
\text { SCOPuS }\end{array}$ & $\begin{array}{c}\text { Sivri, Çolak }{ }^{26} \\
\text { Relato de } \\
\text { Caso }\end{array}$ & $\begin{array}{l}10 \text { anos, diagnóstico de TDAH e palpação cutânea comórbida. Usou } \\
10 \mathrm{mg} / \text { dia de MPH-LM. Após um mês os sintomas de desatenção } \\
\text { diminuíram e houve melhorias significativas no desempenho acadêmico. } \\
\text { Nenhum efeito adverso foi relatado e a palpação da pele também cessou. }\end{array}$ \\
\hline $\begin{array}{c}2017 \\
\text { Turquia } \\
\text { Scopus }\end{array}$ & $\begin{array}{l}\text { Avcil27 }^{27} \\
\text { Relato de } \\
\text { Caso }\end{array}$ & $\begin{array}{l}9 \text { anos, diagnóstico de TDAH. Iniciou } 18 \mathrm{mg} / \text { dia de MPH-OROS e em três } \\
\text { semanas passou a usar } 27 \mathrm{mg} / \text { dia de MPH-OROS. O início do tratamento } \\
\text { foi bem tolerado. Após } 100 \text { dias de uso com } 27 \mathrm{mg} / \text { dia apresentou } \\
\text { sangramento nasal diariamente, o qual foi associado ao uso do MPH. } \\
\text { Após a interrupção do fármaco, o sangramento cessou. Iniciou-se } \\
\text { atomoxetina e houve boa aceitação. }\end{array}$ \\
\hline $\begin{array}{c}2017 \\
\text { Turquia } \\
\text { SCOPuS }\end{array}$ & $\begin{array}{l}\text { Yektas, } \\
\text { Samurcu, } \\
\text { Tufan } 28 \\
\text { Relato de } \\
\text { Caso }\end{array}$ & $\begin{array}{l}7 \text { anos, diagnóstico de TDAH. Iniciou com MPH-LI 20mg/dia. Como efeito } \\
\text { adverso inicial do uso de MPH apresentou insônia. O tratamento foi } \\
\text { alterado para MPH-OROS } 18 \mathrm{mg} / \text { dia, porém apresentou perda de } \\
\text { sobrancelhas e epistaxe, os quais foram associados ao uso de MPH. Com } \\
\text { a interrupção do medicamento os efeitos adversos cessaram. Entretanto, } \\
\text { os problemas acadêmicos retornaram. }\end{array}$ \\
\hline $\begin{array}{c}2015 \\
\text { Itália } \\
\text { scopus }\end{array}$ & $\begin{array}{l}\text { Pozzi, } \\
\text { Bertella, } \\
\text { Molteni, et } \\
\text { al. }{ }^{29} \\
\text { Relato de } \\
\text { Caso }\end{array}$ & $\begin{array}{l}10 \text { anos, com comportamento de oposição intolerável. Usou } 5 \mathrm{mg} / \text { dia, de } \\
\text { MPH-LI e na segunda prescrição usou } 5 \mathrm{mg} \text { duas vezes ao dia. } \\
\text { Inicialmente a medicação foi bem tolerada, a hiperatividade diminuiu e } \\
\text { houve melhora do sono. Após o aumento da dosagem, apresentou } \\
\text { agitação e insônia. O MPH, então, foi descontinuado e os sintomas } \\
\text { desapareceram. Iniciou-se hidroxizina como substituta ao MPH. }\end{array}$ \\
\hline
\end{tabular}


Por consistir em um medicamento estimulante do sistema nervosa central, o metilfenidato foi evidenciado como a medicação de primeira escolha em todos os artigos levantados nesta revisão. Ademais, percebeu-se que a atomoxetina $(29,41 \%)$ foi o fármaco mais utilizado diante da descontinuação do metilfenidato por efeitos colaterais. Observou-se maior frequência na prescrição de MPH LI $(41,2 \%), \mathrm{MPH}$ - OROS (35,3\%) e MPH - LM (23,5\%).

\section{DISCUSSÃO}

O TDAH é o distúrbio neurodesenvolvimental mais comum diagnosticado na infância. Ele consiste em uma doença caracterizada pela presença contínua de desatenção e impulsividade, resultando no comprometimento funcional em diferentes situações, tais como menor desempenho acadêmico e relacionamentos sociais dificultados, além do risco aumentado para hospitalizações, especialmente devido ao uso de substâncias utilizadas para o tratamento do distúrbio ${ }^{30}$. O TDAH se apresenta com maior frequência entre o sexo masculino ${ }^{5}$.

As diretrizes da American Academy of Pediatrics (AAP) para o tratamento do TDHA são direcionadas de acordo com a faixa etária de cada indivíduo ${ }^{30}$. Para crianças em idade pré-escolar, sabe-se que medicações estimulantes podem resultar em instabilidade de humor, portanto, não é recomendado o uso de medicação nessa faixa etária, sendo 
aconselhado a realização de terapia comportamental como medida terapêutica ${ }^{1,30}$.

Dessa forma, destaca-se que a idade dos indivíduos que compõem a amostra pode ser justificada pelo tratamento preconizado diante da doença mais prevalente nos resultados encontrados, O TDHA.

Há relatos da prescrição de metilfenidato para tratar distúrbios do sono, distúrbios ansiosos e depressivos, agitação e para substituição de cocaína, perda de peso e doping, o que não foi evidenciado no presente estudo. A correta prescrição é fundamental para a adesão ao tratamento, pois há relatos de que $61 \%$ dos adolescentes que receberam prescrição de metilfenidato não aderem ao tratamento ${ }^{31}$.

Para crianças em idade escolar, existem evidências de que medicações estimulantes são suficientes no combate aos principais sintomas do TDHA, entretanto, a atomoxetina, guanfacina e clonidina de liberação prolongada, respectivamente, constituem-se como componentes de menor eficácia no tratamento da doença ${ }^{1,5}$.

Observou-se que a maioria dos adolescentes participantes dos estudos encontrados apresentaram melhora rápida dos sintomas do TDHA após a introdução do metilfenidato. Os resultados coadunam com os dados provenientes da AAP, os quais afirmam que, para a maioria dos adolescentes, os estimulantes são altamente efetivos na redução dos principais sintomas ${ }^{1}$. 
Entretanto, chama-se a atenção para os efeitos adversos provocados pelo uso contínuo do metilfenidato, assim como para os resultados observados com a interrupção da medicação. Verificou-se irregularidades de origem física, emocional e comportamental descritas pelo aumento da duração do ciclo menstrual, discinesia orofacial e coreoatetose dos membros, tricotilomania, marcha atáxica, alopecia difusa, sonolência, ansiedade, ejaculação espontânea, pensamentos delirantes, irritabilidade, priapismo, insônia, vitiligo, diminuição do apetite, epistaxe, perda de sobrancelhas, diminuição da contagem das plaquetas e níveis séricos de testosterona e gagueira26,29-31.

Os eventos adversos são uma preocupação significativa entre os pacientes ${ }^{7}$, e a farmacovigilância tem demonstrado essa preocupação com a segurança na utilização do metilfenidato, especialmente em relação aos efeitos adversos, o uso a longo prazo e indevido da medicação, sobretudo diante da expansão do número de prescrições em crianças e adolescentes. Os efeitos adversos mais comuns com a utilização do medicamento foram efeitos neuropsiquiátricos, distúrbios do sistema nervoso (enxaqueca, euforia, distúrbios do sono, acidente vascular cerebral), distúrbios do sistema cardiovascular (hipertensão, distúrbios do ritmo cardíaco), náusea, vômito, diminuição do apetite, perda de peso e fadiga7,31.

Embora a utilização do metilfenidato para o tratamento da TDHA possua uma eficácia conhecida de 75 a $80 \%$ dos casos, estudos mostram que a medicação é conhecida por 
apresentar efeitos adversos comuns como náuseas, dores de estômago, inapetência alimentar, insônia e cefaleia, sendo que movimentos involuntários, disforia e instabilidade de humor são menos frequentes ${ }^{32}$. Tais evidências coadunam com os resultados encontrados, dos quais muitos relatos de casos apontaram alguns dos efeitos adversos supracitados.

\section{CONCLUSÃO}

Essa revisão integrativa levou à identificação de evidências científicas sobre a prescrição, uso e razões para descontinuar o metilfenidato, demonstrando a necessidade de criar estratégias de monitoramento terapêutico que busquem garantir o uso seguro, correto e necessário do medicamento para promover melhor resultado na reabilitação do paciente e na qualidade de vida dos usuários, principalmente no contexto da assistência a crianças e adolescentes.

Além disso, evidenciou-se a escassez de literatura que descreva o manejo adequado do uso do metilfenidato, principalmente no que se refere à participação da enfermagem como protagonista no cuidado de crianças e adolescentes em face da medicalização e aos efeitos adversos que esse fenômeno tem causado.

\section{REFERÊNCIAS}

1. Wolraich ML, Hagan JF, Allan C, Chan E, Davison D, Earls M, et al. Clinical Practice Guideline for the Diagnosis, Evaluation, and Treatment 
of Attention-Deficit/Hyperactivity Disorder in Children and Adolescents. Pediatrics 2019;144:e20192528. https://doi.org/10.1542/peds.20192528

2.Grimmsmann T, Himmel W. The 10 -year trend in drug prescriptions for attention-deficit/hyperactivity disorder (ADHD) in Germany. Eur J Clin Pharmacol 2020;1-9. https://doi.org/10.1007/s00228-02002948-3

3.Speerforck S, Hertel J, Stolzenburg S, Grabe HJ, Carta MG, Angermeyer $\mathrm{MC}$, et al. Attention Deficit Hyperactivity Disorder in Children and Adults: A Population Survey on Public Beliefs. J Attent Dis 2019;00:1-11. https://doi.org/10.1177/1087054719855691

4.Owens J. Social Class, Diagnoses of Attention-Deficit/Hyperactivity Disorder, and Child Well-Being. J Health Social Beh 2020;00:1-17. https://doi.org/10.1177/0022146520924810

5.Boletim Brasileiro de Avaliação de Tecnologias em Saúde. Metilfenidato no tratamento de crianças com transtorno de déficit de atenção e hiperatividade, ano VIII, no. 23 [Internet]. Brasília, 2014 [acesso em: 08 jul 2020]. Disponível em:

https://www.gov.br/anvisa/pt-

br/centraisdeconteudo/publicacoes/regulamentacao/boletim-

brasileiro-de-avaliacao-de-tecnologias-em-saude-brats/boletim-

brasileiro-de-avaliacao-de-tecnologias-em-saude-brats-no-

23.pdf/view

6. Bachmann CJ, Wijlaars LP, Kalverdijk LJ. Trends in ADHD medication use in children and adolescents in five western countries, 20052012. Eur Neuropsychopharmacol 2017;27:484-93.

https://doi.org/10.1016/j. euroneuro.2017.03.002

7.Chen X, Faviez C, Schuck S, Lillo-Le-Louët A, Texier N, Dahamna B, et al. Mining Patients' Narratives in Social Media for Pharmacovigilance: Adverse Effects and Misuse of Methylphenidate. Front Pharmacol 2018;9:541. https://doi.org/10.3389/fphar.2018.00541

8.Storebø OJ, Pedersen N, Ramstad E, Kielsholm ML, Nielsen SS, Krogh $\mathrm{HB}$, et al. Methylphenidate for attention deficit hyperactivity disorder (ADHD) in children and adolescents - assessment of adverse events in non-randomised studies. Cochrane Database Syst Rev 2018; 5:CD012069.

https://doi.org/10.1002/14651858.CD012069.pub2

9.Goode AP, Coeytaux RR, Maslow GR, Davis N, Hill S, Namdari B, et al. Nonpharmacologic Treatments for Attention-Deficit/Hyperactivity Disorder: A Systematic Review. Pediatrics 2018;141:e20180094. https://doi.org/10.1542/peds.2018-0094

10.Sousa LMM, Marques-vieira CMA, Severino SSP, Antunes AV. A metodologia de revisão integrativa da literatura em enfermagem. Rev Invest Enferm 2017;17:17-26.

https://www.researchgate.net/publication/321319742

11.Souza MT, Silva MD, Carvalho R. Revisão integrativa: o que é e como fazer. Einstein (São Paulo) 2010;8:102-6.

https://doi.org/10.1590/s1679-45082010rw1134 
12. Ribeiro RP, Aroni P. Normatização, ética e indicadores bibliométricos em divulgação científica: revisão integrativa. Rev Bras Enferm 2019;72:1723-9. https://doi.org/10.1590/0034-7167-2018-0283

13.Arslan EA, Arslan E, Kilinç A, Göksu Ö. Effect of biperiden treatment in acute orofacial and extremity dyskinesia with methylphenidate therapy. Pediatr Emerg Care 2018;34:e217-8.

https://doi.org/10.1097/PEC.0000000000001102

14.Gunes S. Modified-Release Methylphenidate-Related Trichotillomania in a Boy with Autism Spectrum Disorder. J Child Adol Psychopharmacol 2017;27:675-6.

https://doi.org/10.1089/cap.2017.0001

15.Snell LB, Bakshi D. Neurological adverse effects of methylphenidate may be misdiagnosed as meningoencephalitis. BMJ Case Rep 2015; bcr2014207796. http://dx.doi.org/10.1136/bcr-2014-207796 16. Bilaç Ö, Kütük MÖ, Bilaç C. Hair loss due to methylphenidate use: A case study. Indian J Psychiatry 2018;60:159-60. http://www.indianjpsychiatry.org/text.asp?2018/60/1/159/229957

17.Öncü B, Çolak B, Er O. Methylphenidate-induced spontaneous ejaculation. Ther Adv Psychopharmacol 2015;5:59-61.

https://doi.org/10.1177/2045125314561993

18.Ekinci O, Direk MÇ, Ekinci N, Okuyaz C. Manic symptoms due to methylphenidate use in an adolescent with traumatic brain injury. Clin Psychopharmacol Neurosci 2016;14:314-7.

https://doi.org/10.9758/cpn.2016.14.3.314

19.Ekinci O, Üstündağ B, Tunali BD, Abdulrazzaq Al-Bayati MB, Demir $\mathrm{H}$, Adak İ. Priapism with methylphenidate use in a preschool-aged boy resolved with switching to atomoxetine. Clin Neuropharmacol 2019;42:184-5. https://doi.org/10.1097/WNF.0000000000000357

20.Aksu GG, Akdere P, Toros F. Methylphenidate associated vitiligo in a child: A case report. Asia Pac Psychiatry 2019;11:e12351. https://doi.org/10.1111/appy.12351

21.Ercan ES, Inci SB, Ipci M. Is there an effect of methylphenidate on thrombocytopenia? J Clin Psychopharmacol 2017;37:374. https://doi.org/10.1097/JCP.0000000000000690

22.Akaltun İ. Report of a 14-Year-Old Boy Whose Testosterone Level Decreased After Starting on Methylphenidate. J Child Adolesc Psychopharmacol 2016;26:181.

https://doi.org/10.1089/cap.2015.0209

23.Alpaslan AH, Coşkun KŞ, Kocak U, Gorücü Y. Stuttering associated with the use of short-acting oral methylphenidate. J Clin Psychopharmacol 2015;35:739-41.

https://doi.org/10.1097/JCP.0000000000000403

24. Mutlu C, Bahalı K, Gunes $\mathrm{H}$, Adaletli $\mathrm{H}$. Increase in menstrual cycle length induced by extended-release methylphenidate in an adolescent with attention-deficit/hyperactivity disorder. J Child Adolesc Psychopharmacol 2016;26:860-1.

https://doi.org/10.1089/cap.2015.0131

25. Hu LY, Lin YL, Chang HS, Lu T, Lin WS. Low-dose methylphenidate monotherapy for features of attention-deficit/hyperactivity disorder 
secondary to hereditary cerebellar ataxia. CNS Neurosci Ther 2015;21:672-3. https://doi.org/10.1111/cns.12403

26.Sivri RC, Çolak B. Cessation of skin picking symptoms with methylphenidate treatment in a child with comorbid skin picking and attention-deficit/hyperactivity disorder. Clin Neuropharmacol 2019;42:105-7. https://doi.org/10,1097/wnf.0000000000000338

27. Avcil S. Nasal bleeding probably associated with methylphenidate. J Child Adolesc Psychopharmacol 2017;27:924-5.

https://doi.org/10.1089/cap.2017.0128

28.Yektaş Ç, Samurcu ND, Tufan AE. Loss of eyebrows (madarosis) after use of long-acting methylphenidate: case report. J Clin Psychopharmacol 2017;37:485-6.

https://doi.org/10.1097/JCP.0000000000000713

29.Pozzi M, Bertella S, Molteni M, Antoniazzi S, Carnovale C, Gentili M, et al. Restless sleep in a hyperactive girl: a paradoxical adverse reaction to methylphenidate. J Clin Psychopharmacol 2015;35:738-9. https://doi.org/10.1097/JCP.0000000000000396

30.Danielson ML, Bitsko, RH. Prevalence of Parent-Reported ADHD Diagnosis and Associated Treatment Among U.S. Children and Adolescents, 2016. J Clin Child Adoles Psychol 2018;00:1-14. https://doi.org/10.1080/15374416.2017.1417860

31. Kosse RC, Bouvy ML, Philbert D, de Vries TW, Koster ES. Attentiondeficit/hyperactivity disorder medication use in adolescents: the patient's perspective. J Adolesc Health 2017;61:619-25. https://doi.org/10.1016/j.jadohealth.2017.05.027

32.Ismi O, Yildirim V, Vayisoglu Y, Togrul A, Toros F, Unal M. The effect of methylphenidate on the hearing of children with attention deficit hyperactivity disorder. Inter Arch Otorhinolaryngol 2017;1-5. https://doi.org/10.1055/s-0037-1605367 